Revista Brasileira de Agricultura Irrigada v.10, nº.3, p. 705 - 714, 2016

ISSN 1982-7679 (On-line)

Fortaleza, CE, INOVAGRI - http://www.inovagri.org.br

DOI: $10.7127 /$ rbai.v10n300444

Protocolo 444.16 - 24/05/2016 Aprovado em 15/06/2016

\title{
UTILIZAÇÃO DO MODELO ZAE/FAO PARA A ESTIMATIVA DA PRODUTIVIDADE DO SORGO EM ALAGOAS
}

\author{
Allan Cunha Barros ${ }^{1}$, Remy Farias de Souza ${ }^{2}$, Alexandre Hugo César Barros ${ }^{3}$, José Nildo \\ Tabosa $^{4}$, Antenor de Oliveira Aguiar Netto ${ }^{5}$, Gregório Guirado Faccioli ${ }^{6}$
}

\section{RESUMO}

O manejo adequado, como a escolha da época de plantio, tratos culturais ou o uso da irrigação, permite o maior aproveitamento dos recursos naturais disponíveis no ambiente. Por meio dos modelos pode-se variar os manejos e estimar a produtividade agrícola da cultura do sorgo, permitindo avaliar os diversos tipos de manejos. O objetivo desse trabalho foi calibrar o modelo de zona agroecológica/FAO (ZAE) para estimar a produtividade e avaliar as épocas de plantio de menor risco para a cultura do sorgo no Estado de Alagoas. Para calibração do modelo ZAE/FAO foram utilizados dados de produtividade, provenientes do Programa de Melhoramento Genético de Sorgo do Nordeste na região de Araripina - PE, conduzido pelo Instituto de Pesquisa Agropecuária (IPA) e pela Embrapa. Após a calibração foram simulados os cenários para as cidades de Água Branca, Arapiraca, Palmeira dos Índios e Porto de Pedras, em Alagoas, baseados em épocas de plantios de 10 em 10 dias, e para CAD do solo de 30, 40 e 65 mm. As produtividades variaram de 1,8 a 1,9 Mg ha-1 ${ }^{1}$ em Água Branca, de 3,7 a 4,1 Mg ha1 em Arapiraca, 1,9 a 2,0 Mg ha- ${ }^{1}$ em Palmeira dos Índios, 2,2 a 2,3 Mg ha-1 em Porto de Pedras, no mês de plantio mais produtivo.

Palavra-Chave: modelagem matemática, disponibilidade hídrica, ISNA

\section{USE OF MODEL ZAE / FAO FOR ESTIMATING PRODUCTIVITY OF SORGHUM IN ALAGOAS}

\footnotetext{
${ }^{1}$ Eng. Agrônomo. Professor do Curso de Agronomia da UFAL, Campus Arapiraca, Av. Manoel Severino Barbosa, Bom Sucesso, Arapiraca, AL, Brasil. email: allan.cunha.barros@gmail.com

${ }^{2}$ Eng. Agrônomo. Mestre em Agricultura e Ambiente. Av. Manoel Severino Barbosa, Bom Sucesso, Arapiraca, AL, Brasil. email: remyfarias@hotmail.com

${ }^{3}$ Eng. Agrônomo. Pesquisador da Embrapa Solos, R. Antônio Falcão, 402 - Boa Viagem, Recife, PE, Brasil. email: alex.hugo.2006@gmail.com

${ }^{4}$ Eng. Agrônomo. Pesquisador Instituto Agronômico de Pernambuco, AV. Gal San Martin, 1371

Bongi, Recife, PE, Brasil. email: nildo.tabosa@ipa.br

${ }^{5}$ Eng. Agrônomo. Professor do Curso de Agronomia da UFS, Departamento de Eng. Agronômica, Av. Marechal Rondon, S/n - Jardim Rosa Elze, São Cristóvão, SE, Brasil. email: antenor.ufs@gmail.com

${ }^{6}$ Eng. Agrícola. Professor do Curso de Eng. Agrícola da UFS, Departamento de Eng. Agrícola, Av. Marechal

Rondon, S/n - Jardim Rosa Elze, São Cristóvão, SE, Brasil. email: gregorioufs@gmail.com

${ }^{6}$ Estudante de Agronomia Arapiraca - Alagoas. Email: klinger.nunes@gmail.com
} 


\begin{abstract}
The proper management, the choice of planting time, cultivation or use of irrigation, allows greater use of natural resources available in the environment. Through the models can vary the managements and estimate the agricultural productivity of sorghum in order to evaluate the various types of managements. . Thus it is possible to know the crop response to water availability of each site and its variation throughout the year. The aim of this study was to calibrate the agroecological zone model of FAO (AEZ), estimate the productivity and assess which lower risk of planting dates for the sorghum crop in the State of Alagoas. For calibration of the ZAE / FAO model were used productivity data from the Breeding Program of Northeast Sorghum in Araripina- PE, conducted by the Instituto de Pesquisa Agropecuária (IPA) and Embrapa. After calibration were simulated scenarios for the cities: Água Branca, Arapiraca, Palmeira dos Índios and Porto de Pedras, Alagoas, based on 10 plantations times in 10 days, and soil CAD for 30, 40 and $65 \mathrm{~mm}$.. The model presented adjustment $\mathrm{R}^{2}=0.87$; Wilmont index $(\mathrm{d})=0.94$ and reliability index $(\mathrm{CI})$ of 0.87 . Yields ranged from 1.8 to $1.9 \mathrm{Mg}^{\mathrm{Ma}}{ }^{1}$ in Água Branca, 3.7 to $4.1 \mathrm{Mg}^{\mathrm{ha}}{ }^{1}$ in Arapiraca, 1.9 to $2.0 \mathrm{Mg}^{-1}{ }^{-1}$ in Palmeira dos Indios , 2.2 to $2.3 \mathrm{Mg}_{\text {ha- }}{ }^{1}$ in Porto de Pedras, in the month of planting more productive.
\end{abstract}

Keywords: mathematical modeling, water availability, ISNA

\section{INTRODUÇÃO}

O sorgo (Sorghum bicolor) é uma planta de origem tropical, bem adaptada a regiões áridas e semiáridas, exigindo clima quente para poder expressar o seu potencial produtivo. A cultura, com características xerófilas, é considerada tolerante a períodos secos, notadamente em regiões do Nordeste do Brasil. $\mathrm{Na}$ região Nordeste, o cultivo do sorgo é realizado durante a estação chuvosa, cujo período é curto e com distribuição irregular, caracterizado, ainda, por ocorrência de veranicos, com 15 a 20 dias sem chuvas. Em Pernambuco, as principais regiões produtoras localizam-se no Agreste e no Sertão (TABOSA, 2012).

A cultura do sorgo exige em torno de 300 $\mathrm{mm}$ a $400 \mathrm{~mm}$ de precipitação pluviométrica, distribuídos regularmente durante o seu ciclo de crescimento e desenvolvimento para que se alcancem níveis de produtividade satisfatórios. As fases fenológicas críticas da cultura, quanto à deficiência hídrica, correspondem ao estádio de plântula e no florescimento, sendo imprescindível nessas épocas um adequado nível de suprimento de água para uma boa produção (TABOSA et al. 2008).A temperatura ótima para o desenvolvimento da cultura varia conforme a cultivar considerada.
De modo geral, temperaturas do ar superiores a $38^{\circ} \mathrm{C}$ ou inferiores a $16{ }^{\circ} \mathrm{C}$ limitam o desenvolvimento da maioria das cultivares.

O sorgo apresenta elevado potencial para ser utilizado na alimentação de animais, sobretudo nas regiões semiáridas, devido às características de resistência à deficiência hídrica (OLIVEIRA et al.2002). Contudo, a produtividade média é considerada baixa no Nordeste, em torno de $3.128 \mathrm{~kg} \mathrm{ha}^{-1}$ (IBGE, 2013), principalmente devido à falta de manejo adequado, com relação a pouca aplicação de fertilizantes, a densidade de plantio inadequada e a irregularidade espaço-temporal da precipitação pluviométrica(SANTOS et al. 2013).

Nesse sentido, a estimativa da produtividade para a cultura do sorgo, utilizando o modelo de Zona Agroecológica (ZAE) da FAO (DOORENBOS;KASSAM, 1979) pode ajudar no planejamento agrícola de localidades no Nordeste Brasileiro. O modelo de ZAE é um dos mais utilizados em estudos para estimar a produtividade agrícola de culturas (ASSAD et al. 2007; ANDRIOLLI;SENTELHAS, 2009).

Souza et al. (2014) utilizaram o modelo ZAE para estimar a produtividade do milho no Estado de Alagoas, em condições irrigadas e 
não irrigadas, demonstrando o potencial da ferramenta para a utilização no Estado.

Visando um melhor aproveitamento dos recursos hídricos e o conhecimento das melhores épocas de se produzir o sorgo em Alagoas, desenvolveu-se esse trabalho com o objetivo de calibrar o modelo ZAE, para estimar a produtividade e avaliar quais as épocas de plantio com menor risco para a semeadura da cultura do sorgo no Estado de Alagoas.

\section{MATERIAL E MÉTODOS}

O estudo foi desenvolvimento na Universidade Federal de Alagoas, Campus Arapiraca em parceria como Instituto Agronômico de Pernambuco - IPA e a Embrapa Solos, UEP Recife, durante o ano de 2013.
O projeto foi divido em duas partes, na primeira foi feita a calibração do modelo para a cultura do sorgo, na segunda, foram feitas simulações de produtividade do sorgo para os municípios de Alagoas.

\section{Descrição do modelo}

O modelo da Zona Agroecológica (ZAE) estima a produtividade máxima da cultura, também chamada de produtividade potencial (PP) (DOORENBOS; KASSAM, 1979; BATTISTI, 2013), cujos conceitos seguem os princípios de De Wit (1965). O modelo é calculado em duas etapas: a) estimativa da produtividade máxima ou potencial (Ymp) e b) a penalização da produtividade potencial, devido à deficiência hídrica da cultura, obtendo-se a produtividade real ou atingível (Yr).

$$
\mathrm{Ymp}=\mathrm{cL} \times \mathrm{cN} \times \mathrm{cH} \times \mathrm{G}[\mathrm{F}(\mathrm{a}+\mathrm{b} \times \mathrm{ym}) \mathrm{yo}+(1-\mathrm{F})(\mathrm{c}+\mathrm{d} \times \mathrm{ym}) \mathrm{yc}]
$$

Em que:

Ymp - Produtividade potencial máxima da cultura (kg.ha-1.período ${ }^{-1}$ ); cL - correção em função do desenvolvimento da cultura e área foliar; cN - correção para a produção de matéria seca; cH - correção para a parte colhida; $\mathrm{G}$ - período total do crescimento (dia); F - Fração do dia em que o sol fica encoberto por nuvens; Ym - Taxa de produção de matéria seca das folhas (kg.ha-1 ${ }^{1}$.período-1 ${ }^{1}$; a, b, c e d Coeficientes da equação (tabelados); Yc - taxa de produção bruta de matéria seca (kg.ha${ }^{1}$.período ${ }^{-1}$ ).

A produtividade real (Yr) é calculada por meio da correlação do crescimento da cultura com o consumo de água. $\mathrm{O}$ fator de sensibilidadedevido ao déficit hídrico (Ky) é calculado de acordo com as fases fenológicas da cultura. Para a estimativa da produtividade real (Yr), o modelo ZAE utiliza a produtividade potencial máxima da cultura (Ymp), o coeficiente de resposta da cultura, o total da evapotranspiração real e o total da evapotranspiração máxima da cultura durante seu ciclo (OLIVEIRA et al., 2010). Dessa forma, calcula-se o Yr da seguinte forma (Eq. 2):

$$
Y r=\operatorname{Ymp}\left[1-k y\left(1-\frac{E T r}{E T p}\right)\right]
$$

em que, Ky é o coeficiente empírico, que representa a sensibilidade da cultura a deficiência hídrica (-);ETr é a evapotranspiração real da cultura $\left(\mathrm{mm} \mathrm{d}^{-1}\right)$; e ETp é a evapotranspiração máxima da cultura $\left(\mathrm{mm} \mathrm{d}^{-1}\right)$. O cálculo da ETp é realizado por meio da seguinte equação (3):

$$
E T p=E T o \times K c
$$

em que, ETp é a evapotranspiração máxima da cultura $\left(\mathrm{mmd}^{-1}\right)$; ETo é a evapotranspiração de referência $\left(\mathrm{mmd}^{-1}\right)$; e Kc o coeficiente de cultura. A ETo foi calculada de acordo com Penman-Moneith (ALLEN et al. 1998). Os valores de Kc utilizados nas fases fenológicas foram: fase I $(0,4)$; fase II $(1,1)$; fase III $(0,88)$; 
e fase IV $(0,58)$.Para a determinação da ETr utilizou-se o coeficiente de estresse hídrico (Ks) que penaliza o consumo de água da planta em função do déficit hídrico do solo (Eq.4):

$$
K s=\frac{\ln [L A A+1]}{\ln [C T A+1]}
$$

em que, LAA é a lâmina de água no solo (mm); CTA é a capacidade total de água no solo (mm). Assim, ETr é calculado por meio da equação:

$$
E T r=E T o \times K c \times K s
$$

\section{Calibração}

Para a calibração do modelo foram utilizados dados de produtividade do sorgo na região de Araripina - PE (lat. 07³4'34”S, long. 40²9'54”'W, altitude de 622m), durante um período de quinze anos (1988 e 1899, 1991 a 2003),coletados do Programa de Melhoramento Genético de Sorgo do Nordeste da Embrapa/IPA. Os dados foram os mesmos utilizados por Barros (2010) e Martins (2012), que utilizaram modelos para prever a produtividade da cultura do sorgo nessas regiões.

O ciclo da cultura foi estabelecido em 129 dias, compreendendo quatro fases fenológicas: $1^{\mathrm{a}}$ fase, 22 dias, da emergência até a diferenciação; $2^{\mathrm{a}}$ fase, 36 dias, reprodutiva; $3^{\text {a }}$ fase, 42 dias, do emborrachamento à floração; e a 4 a fase, 29 dias, da fase leitosa a maturação fisiológica (MAGALHÃES et al. 2000).

Os valores utilizados para as correções do modelo foram provenientes da literatura sobre a fisiologia da cultura do sorgo (EMBRAPA, 2013), os quais foram adaptados de acordo com as calibrações realizadas para a região de Araripina, PE. Adotou-se como profundidade do sistema radicular da cultura como $0,15 \mathrm{~m}$ para as fases I e II; $0,45 \mathrm{~m}$ e 0,5 $\mathrm{m}$, para as fases III e IV, respectivamente.

Os dados de solo foram coletados de Barros (2010) e consistiu basicamente dos valores de Capacidade de Campo e Ponto de
Murcha (à base volume). Dados climatológicos foram cedidos pelo Instituto Agronômico de Pernambuco - IPA, da estação experimental de Araripina.

As variáveis utilizadas na calibração foram a cL - correção em função do desenvolvimento da cultura e área foliar; $\mathrm{cN}$ correção para a produção de matéria seca; cH - correção para a parte colhida. Utilizou-se o procedimento de tentativa e erro por análise visual.

A avaliação do desempenho da estimativa da produtividade da cultura do sorgo foi avaliada utilizados os seguintes índices estatísticos: coeficiente de determinação $\left(\mathrm{R}^{2}\right)$, o índice de Willmott (d) e o índice de concordância (IC), conforme Camargo e Sentelhas (1997).

\section{Cenários simulados}

Segundo Mantovani (1993), os modelos são mais exigentes nos dados necessários, porém permitem a transferibilidade e aplicabilidade para outras condições. Como os modelos possuem como princípio básico sua utilização para diversas regiões, e não se dispunha de dados de produtividade para a região de Alagoas, , considerou-se a calibração do modelo feita para Araripina-PE, como válida para a utilização nas simulações dos cenários em Alagoas.Ressalta-se que nos dados tabelados do modelo ZAE não houve diferença entre a latitude $0^{\circ}$ até $10^{\circ}$. Mesmo procedimento utilizado por Souza et al. (2014) para a cultura do milho.

Os cenários da simulação foram baseados nas épocas de plantio e na disponibilidade de água para a cultura. Assumiu-se três épocas de plantio por mês (os dias 10, 20 e 30, de cada mês) $X$ Disponibilidade de água (3 CADS: 30mm CAD 1, 40mm CAD 2 e 65mm CAD 3), totalizando 108 cenários.

As produtividades simuladas foram para as cidades alagoanas de Arapiraca (0945’09”S, 36³9’40”W, 264m), Água Branca(09¹5'39”S, 3756’10”W, 570m), Palmeira dos Índios (09 $24^{\prime} 26^{\prime \prime} \mathrm{S}$, 36³7'39”'W, 342m) e Porto de Pedras (0909'30”'S, 35¹7’42”'W, 22m), cujos dados 
climatológicos foram cedidos pelo o Instituto Nacional de Meteorologia - INMET.

\section{RESULTADOS E DISCUSSÃO}

A diferença média entre a produtividade da cultura do sorgo observada e estimada foi da ordem de $\pm 183 \mathrm{~kg} \mathrm{ha}^{-1}$, a mesma ordem de grandeza também é verificada entre os valores. A estimativa de produtividade superestimou os valores observados em 40\%dos anos (6 anos) e subestimou em $60 \%$ (9 anos), com erro médio de $818 \mathrm{~kg} \mathrm{ha}^{-1}$. Apenas no ano de 1993 que a produtividade observada atingiu um valor muito inferior a média de $3.467 \mathrm{~kg} \mathrm{ha}^{-1}$ (Tabela
1). É provável que esta produtividade tenha ocorrido devido à ocorrência do forte efeito do El Niño em 1993 (Martins, 2011).

Como o modelo da Zona Agroecológica utiliza parâmetros de cultura, como o coeficiente de sensibilidade a deficiência hídrica (Ky) e o coeficiente de colheita (cH), esses coeficientes são sensíveis a variações climáticas, principalmente a eventos extremos como a ocorrência de eventos El-NiñoOscilação Sul (ENOS). Observa-se que os anos de fortes e moderados de El-Niño (1992/1993, 1997/1998 e 2001/2002), a diferença entre os valores estimados e observados de produtividade tendem a serem maiores (Tabela 1).

Tabela 1. Produtividade da cultura do sorgo observada e estimada para localidade de Ararpina, PE.

\begin{tabular}{lrrr}
\hline Ano & $\begin{array}{r}\text { Observado } \\
\text { Produtividade (Mg ha- }\end{array}$ & $\begin{array}{c}\text { Estimado } \\
\text { Piferença }\end{array}$ \\
\hline 1988 & 5297 & 4631 & 666 \\
1989 & 5093 & 4441 & 652 \\
1991 & 4079 & 3946 & 133 \\
1992 & 3270 & 3184 & 86 \\
1993 & 700 & 2471 & -1771 \\
1994 & 4300 & 3922 & 378 \\
1995 & 4600 & 4749 & -149 \\
1996 & 4786 & 4516 & 270 \\
1997 & 3513 & 3616 & -103 \\
1998 & 3020 & 3725 & -705 \\
1999 & 3623 & 3885 & -262 \\
2000 & 2485 & 3146 & -661 \\
2001 & 2363 & 2587 & -224 \\
2002 & 1557 & 2543 & -986 \\
2003 & 3317 & 3386 & -69 \\
\hline Media & $\mathbf{3 4 6 7}$ & $\mathbf{3 6 5 0}$ & $\mathbf{- 1 8 3}$ \\
\hline
\end{tabular}

O modelo ZAE foi testado em praticamente todas as regiões no Brasil, utilizando diversas culturas agrícolas, com resultados que auxiliam ao produtor minimizar as perdas de produtividade decorrentes do estresse hídrico (ROLIM et al. 2001; BONNECARRÈRE et al. 2007; ANDRIOLI; SENTELHAS, 2009; SHRESTHA et al., 2010).

Para a localidade de Araripina, PE o índice de Willmott foi de 0,93 e o de concordância (IC) 0,87 (ótimo), evidenciando o potencial do modelo ZAE para ser utilizado em diferentes estudos, devido à sua simplicidade, facilidade de ajuste e aplicabilidade (Figura 1). Verifica-se, ainda, pouca dispersão entre os dados de produtividade observados e estimados (reta 1:1) (Figura 1). Nesse sentido, quando se analisa a proporção do erro médio de $818 \mathrm{~kg}$ $\mathrm{ha}^{-1}$ na estimativa da produtividade, por meio das barras de erros, observa-se que a maioria dos valores estimados estão dentro do intervalo permitido (Figura 2). 


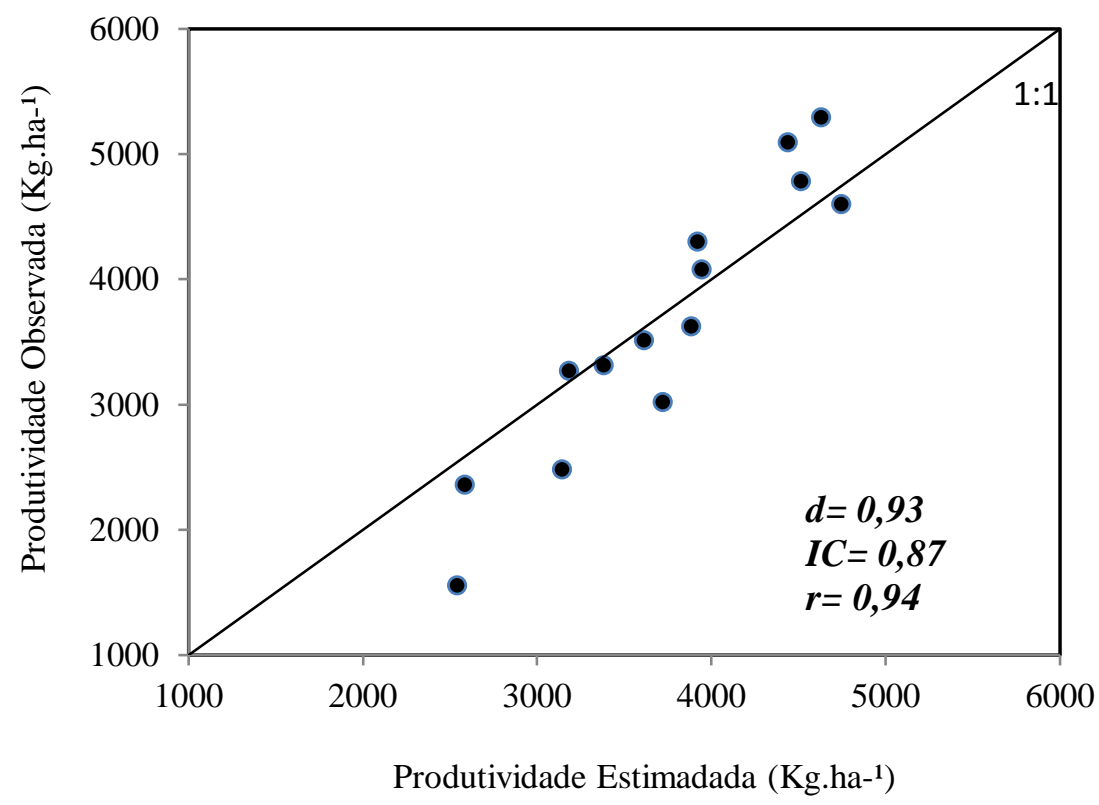

Figura 1. Relação entre produtividade observada e estimada com o modelo da Zona Agroecológica - FAO para cultura da sorgo, em Araripia, PE

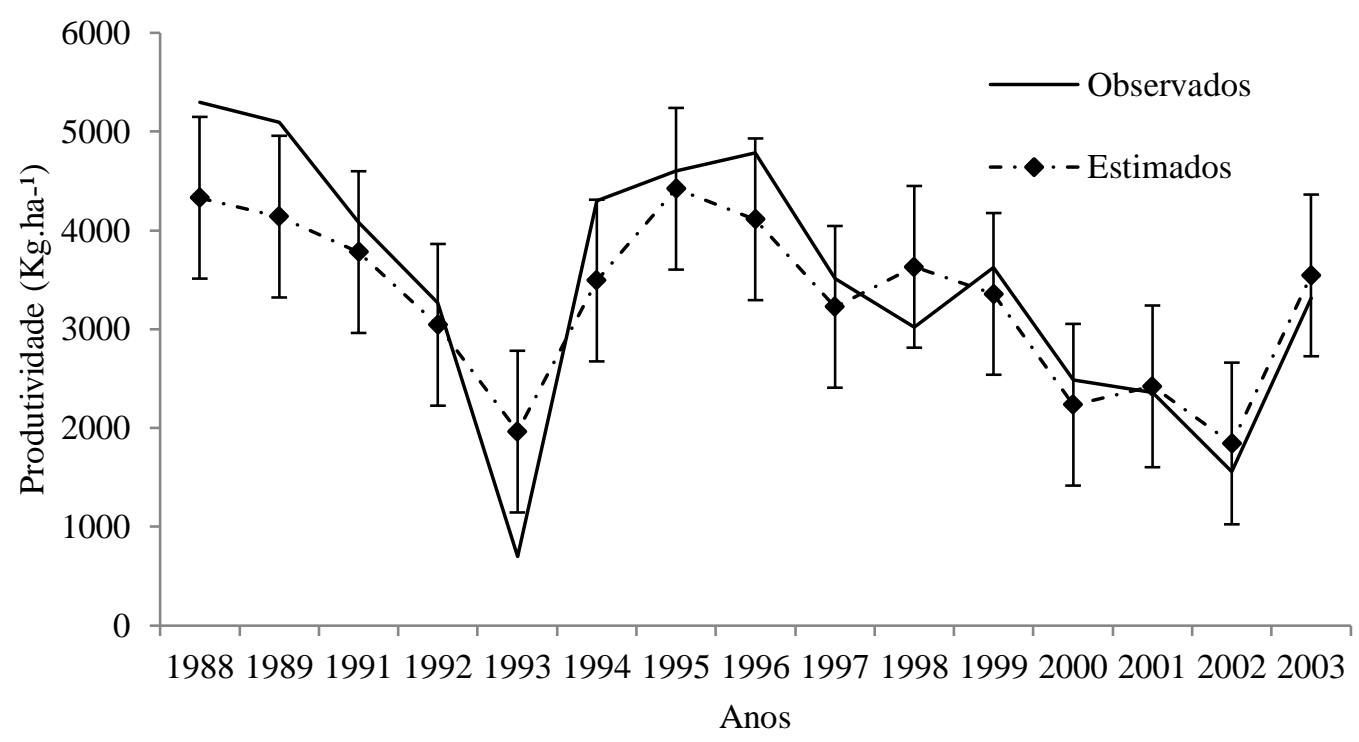

Figura 2. Erro médio entre produtividade observada e estimada com o modelo da Zona Agroecológica - FAO para cultura da sorgo.

O índice de concordância (IC) demonstrou resultado ótimo, próximo aos encontrados por Martin (2012) no município de Araripina, PE, utilizando os modelos de Zona Agroecológica e de Wageningen. Segundo Sentelhas (2011) vários aspectos devem ser considerados ao escolher o modelo de balanço hídrico para o monitoramento da produtividade agrícola, pois a principal limitação no uso de modelos mais complexos é o grande número e a complexidade das variáveis de entrada. De fato, muitas vezes os dados necessários para uma avaliação mais completa não estão disponíveis, tornado o modelo ZAE uma ferramenta indispensável para avaliação de riscos na agricultura, principalmente em regiões, onde a variabilidade da precipitação pluviométrica é bastante acentuada, como a região do Nordeste do Brasil.

\section{Cenários Simulados}


$\mathrm{Na}$ Figura 3 estão as produtividades para os municípios de Alagoas simulados com a CAD de 65, 45mm e $30 \mathrm{~mm}$. Observou-se em todos os municípios a mesma tendência. As curvas de produtividade possuem uma similaridade de acordo com o regime hídrico local. Alves et al. (2011) relacionam esse comportamento devido a uma redução da disponibilidade hídrica, com a queda de produtividade devido a redução do número de sementes na panícula.

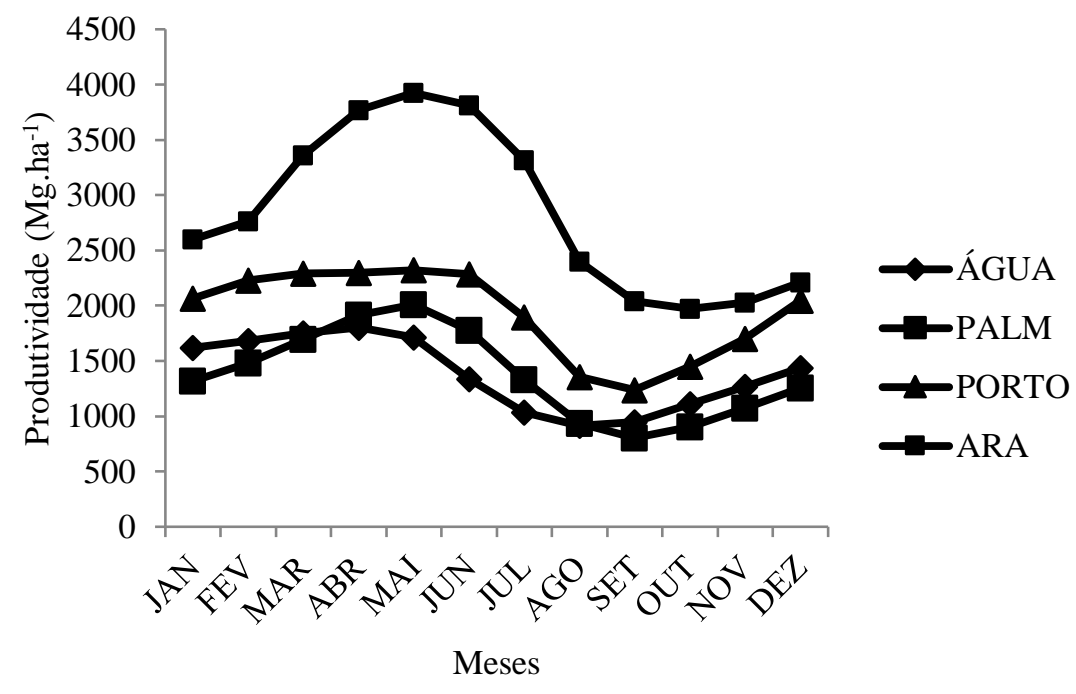

Figura 3. Produtividades em diferentes épocas do ano, para os municípios de Água Branca (ÁGUA), Arapiraca (ARA), Palmeira do Índios (PALM) e Porto de Pedras (PORTO), em Alagoas, com CAD de 65 mm.

Os municípios de Porto das Pedras (PORTO) e Água Branca (ÁGUA) possuem menor inclinação das curvas, demonstrando que ocorre menor variação de temperatura na região durante o ano.

Observa-se que a cidade de Arapiraca obteve as maiores produtividades eapresenta uma relação direta com a maior disponibilidade hídrica local. Já as regiões de Palmeira dos Índios (PALM) e Água Branca (ÁGUA) as menores. Assis et al. (2011) obtiveram exatamente esse comportamento para a condição de sequeiro, onde a disponibilidade hídrica respondeu por $61,9 \%$ da variação total da produtividade, enquanto que os $38,1 \%$ restantes podem estar relacionados a fatores como data de plantio, ano de plantio, radiação solar e temperatura. As épocas de maior produtividade foram para os meses com plantio entre abril a junho, em ARA e PAI, de janeiro a junho em Porto das pedras (PORTO) e para a cidade de Água Branca (ÁGUA), os meses foram entre março a maio.

A variação da CAD, como já esperado, proporcionou redução das produtividades (Tabela 2), apesar da redução das capacidades de armazenamento de aproximadamente 38\% da CAD 1 para a 2, e de $53 \%$ para a 3, as produtividades não acompanharam tão acentuadamente as reduções das CADs.

Tabela 2.Produtividades em diferentes épocas do ano, para os municípios de Água Branca, Arapiraca, Palmeira do Índios e Porto de Pedras, em Alagoas, para a CAD de 65 mm, e redução percentual para a CAD 2 (45mm) e 3 (30 mm).

\begin{tabular}{ccccccc}
\hline & \multicolumn{3}{c}{ Água Branca } & \multicolumn{3}{c}{ Arapiraca } \\
& CAD 1 & CAD 2 & CAD 3 & CAD 1 & CAD 2 & CAD 3 \\
& Kg.ha $^{-1}$ & $\%$ & $\%$ & Kg.ha $^{-1}$ & $\%$ & $\%$ \\
\hline Jan & 1826.1 & -11.5 & -17.9 & 3017.2 & -11.6 & -17.7
\end{tabular}


Barros et al.

\begin{tabular}{|c|c|c|c|c|c|c|}
\hline Fev & 1876.9 & -8.4 & -13.6 & 3197.9 & -9.4 & -14.3 \\
\hline Mar & 1935.1 & -6.3 & -10.3 & 3789.0 & -5.7 & -9.7 \\
\hline Abr & 1921.5 & -5.9 & -9.7 & 3952.9 & -4.9 & -8.1 \\
\hline Mai & 1851.1 & -8.8 & -14.1 & 4108.8 & -5.1 & -9.0 \\
\hline Jun & 1551.9 & -12.6 & -19.2 & 4106.8 & -7.2 & -11.9 \\
\hline Jul & 1453.2 & -12.3 & -18.6 & 3915.5 & -9.1 & -15.1 \\
\hline Ago & 1205.8 & -16.1 & -23.8 & 3226.1 & -10.1 & -16.9 \\
\hline Set & 1236.5 & -16.3 & -24.1 & 2445.8 & -17.4 & -25.4 \\
\hline Out & 1348.2 & -15.6 & -23.3 & 2413.3 & -14.1 & -20.7 \\
\hline Nov & 1524.2 & -14.7 & -21.9 & 2601.8 & -14.4 & -22.0 \\
\hline Dez & 1689.1 & -13.4 & -20.5 & 2720.8 & -11.4 & -17.6 \\
\hline Média & 1618.3 & 1435.4 & 1337.0 & 3291.3 & 2983.1 & 1295.7 \\
\hline Desvio & 268.1 & 292.0 & 296.3 & 659.4 & 717.4 & 384.5 \\
\hline \multirow[t]{4}{*}{$\mathrm{CV}$} & 16.6 & 20.3 & 22.2 & 20.0 & 24.0 & 29.7 \\
\hline & \multicolumn{3}{|c|}{ Palmeira dos Índios } & \multicolumn{3}{|c|}{ Porto de Pedras } \\
\hline & CAD 1 & CAD 2 & CAD 3 & CAD 1 & CAD 2 & CAD 3 \\
\hline & Kg.ha ${ }^{-1}$ & $\%$ & $\%$ & Kg.ha ${ }^{-1}$ & $\%$ & $\%$ \\
\hline Jan & 1493.9 & -11.6 & -17.1 & 2310.5 & -7.9 & -13.4 \\
\hline Fev & 1673.6 & -9.5 & -14.3 & 2400.6 & -5.1 & -9.1 \\
\hline Mar & 1847.3 & -7.4 & -11.2 & 2368.4 & -2.7 & -4.9 \\
\hline Abr & 2002.7 & -4.7 & -7.7 & 2327.7 & -1.4 & -2.8 \\
\hline Mai & 2063.5 & -4.5 & -7.6 & 2346.6 & -1.5 & -2.9 \\
\hline Jun & 1853.2 & -8.7 & -13.6 & 2379.2 & -5.1 & -9.0 \\
\hline Jul & 1474.6 & -10.9 & -16.2 & 2118.7 & -9.1 & -13.7 \\
\hline Ago & 1125.8 & -13.2 & -19.6 & 1676.8 & -12.0 & -17.4 \\
\hline Set & 1103.7 & -16.0 & -23.2 & 1621.4 & -14.2 & -20.4 \\
\hline Out & 1103.7 & -16.0 & -23.2 & 1804.7 & -13.1 & -19.3 \\
\hline Nov & 1230.7 & -14.7 & -21.4 & 2038.3 & -12.3 & -18.6 \\
\hline Dez & 1333.4 & -13.3 & -19.3 & 2255.7 & -9.5 & -15.3 \\
\hline Média & 1525.5 & 1372.7 & 1295.7 & 2137.4 & 1980.3 & 1889.1 \\
\hline Desvio & 355.9 & 380.4 & 384.5 & 286.7 & 346.0 & 363.5 \\
\hline $\mathrm{CV}$ & 23.3 & 27.7 & 29.7 & 13.4 & 17.5 & 19.2 \\
\hline
\end{tabular}

$\mathrm{Na}$ cidade de Arapiraca (ARA) as reduções de produtividade ocorreram principalmente nos meses com menor disponibilidade hídrica, com início de produção em julho. Vale ressaltar que o período de plantio em julho tem deficiência hídrica maior nos meses seguintes, principalmente, de setembro e outubro, época relacionada a fase de formação e enchimento dos grãos, uma vez que ocorre a redução na absorção de nutrientes e na área foliar, acarretando abaixamento na produção de fotoassimilados (ALVES et al., 2011). A redução foi em média 10,0\% para a CAD 2 e
15,7\% para a CAD 3. As maiores reduções ocorreram para o mês de plantio em setembro e em todas as cidades.

A cidade de Porto de Pedras (PORTO) obteve menor redução da produtividade em função da redução da CAD 1, em 7,8 e 12,2 \%, CAD 2 e 3, respectivamente. Pelo fato de Palmeira dos Índios (PALM) ser mais próximo a Arapiraca, obtiveran semelhança entre o período de maior redução de produtividade.

Em Água Branca (ÁGUA) foram observadas as maiores reduções e, tendocomo o principal fator envolvido, a baixa disponibilidade hídrica local, devido a 
localização da cidade está inserida no sertão de Alagoas, apresentando precipitações médias anuais inferiores a $450 \mathrm{~mm}$ (CPRM, 2005). Mesmo que o sorgo necessite de menos água para conversão em matéria seca, quando comparado ao milho e ao trigo, essas precipitações muitas das vezes nas regiões sertanejas ocorrem em um período considerado curto ao ciclo da cultura, apresentando escassez hídricano florescimento, fase crítica da cultura (MAGALHÃES et al, 2000). A média de redução da produtividade na CAD 2 foi de $11,8 \%$ e de $18,1 \%$ para CAD 3.

\section{CONCLUSÕES}

1. A calibração do modelo da Zona Agroecológica - ZAE apresentou bons índices estatísticos para avaliação da produtividade local de sorgo, com índice de concordância (IC) da ordem de 0,87 ;

2. As produtividades médias para a cultura do sorgo foram de 2523, 1463, 1397 e 2002 Mg.ha ${ }^{-1}$ para Água Branca, Arapiraca, Palmeira do Índios e Porto de Pedras, respectivamente.

\section{AGRADECIMENTOS}

Ao Instituto Nacional de meteorologia (INMET), ao Centro Nacional de Pesquisa de Solos UEP Recife da Empresa Brasileira de Pesquisa Agropecuária (EMBRAPA), e o Instituto Agronômico de Pernambuco - IPA, pelos dados edafoclimáticos e da cultura e Fundação de Amparo à Pesquisa do Estado de Alagoas pelo suporte financeiro.

\section{REFERÊNCIAS}

ALLEN, R. G.; PEREIRA, L. S; RAES, D.; SMITH, M. Crop evapotranspiration guidelines for computing crop water requirements. (Irrigation and Drainage Paper, 56). Rome: FAO, 1998.
ALVES, M.E. B..; ANDRADE, C.L.T.; CÁRDENAS, R.R..; AMARAL, T.A.; SILVA, D.F.Identificação e quantificação do efeito de fatores ambientais na produtividade da cultura do milho na região de Janaúba, MG. Revista Brasileira de Agricultura Irrigada, v. 5, nº 3, p. 188 - 201, 2011.

ANDRIOLI, K.G.; SENTELHAS, P.C. Brazilian maize genotypes sensitivity to water deficit estimated through a simple crop yield model. Pesquisa Agropecuária Brasileira, v. 44, n.7, p. 653-660, 2009.

ASSAD, E.D.; MARIN, F.R.; EVANGELISTA， S.R.; PILAU， F.G.; FARIAS, J.R.B.; PINTO, H.S.; JÚNIOR, J.Z. Sistema de previsão de safra para o Brasil. Pesquisa Agropecuária Brasileira, v. 42, p. 615-625, 2007

BARROS, H.C.B. Desenvolvimento de funções de pedotransferência e sua utilização em modelo agro-hidrológico. 2010. 148 p.Tese (Doutorado em Ciências). Escola Superior de Agricultura "Luiz de Queiroz”. Universidade de São Paulo. Piracicaba, SP. 2010.

BONNECARRÈRE, R.A.G.; DOURADONETO, D.; MARTIN, T.N.; PEREIRA, A.R.; MANFRON, P.A. Estimativa das produtividades potencial e deplecionada da cultura do milho no Estado do Rio Grande do Sul em função das condições climáticas. Revista Brasileira de Agrometeorologia, v. 15, n.3, p. 280-288, 2007.

CAMARGO, A.P.; SENTELHAS, P.C. Avaliação do desempenho de diferentes métodos de estimativa da evapotranspiração potencial no estado de São Paulo, Brasil. Revista Brasileira de Agrometeorologia, v. 5, n. 6, p. 89-97, 1997

DE WIT, C.T. Photosynthesis of leaf canopies. Wageningen: PUDOC, 1965. 57 p. (Agriculture Research Report, 663). 
DOORENBOS, J.; KASSAM, A.M. Yield response to water. Rome: FAO, 1979. 300 p. (Irrigation and Drainage Paper, 33).

EMBRAPA- Empresa Brasileira de Pesquisa Agropecuária. Disponível em: $<$ http://sistemasdeproducao.cnptia.embrapa.br /FontesHTML/Sorgo/CultivodoSorgo_4ed/cli ma.htm>. Acesso em 04 de dezembro 2013.

INSTITUTO BRASILEIRO DE GEOGRAFIA E ESTATÍSTICA - IBGE. Disponível em: < http://www.sidra.ibge.gov.br/bda/agric/defaul t.asp? $\mathrm{z}=\mathrm{t} \& \mathrm{o}=11 \& \mathrm{i}=\mathrm{P}>$. Acesso em: 15 dezembro de 2013.

MAGALHÃES, P. C.; DURÂES, F.O.M.; SHAFFERT, R.E. Fisiologia da planta do sorgo. Embrapa milho e sorgo. Circular técnico, 3, 46p. 2000.

MARTINS, M. A. Estimativa de produtividade das culturas do milho e do sorgo a partir de Modelos Agrometeorológicos em algumas Localidades da Região Nordeste do Brasil. 2012. 127 p. Dissertação (Mestrado em Mestrado em Meteorologia). Instituto Nacional de Pesquisas Espaciais. São José dos Campos, SP.

OLIVEIRA, J. S.; FERREIRA, R. P.; CRUZ, C. D.; PEREIRA, A. V.; BOTREL, M. A.; VON PINHO, R. G.; RODRIGUES, J. A. S.; LOPES, F. C. F.; MIRANDA, J. E. C. Adaptabilidade e estabilidade em cultivares de sorgo. Revista Brasileira de Zootecnia, v.31, n.2, p.883-889, 2002.

ROLIM, G.S.; SENTELHAS, P.C.; UNGARO, M.R.G. Análise de risco climático para a cultura de girassol, em algumas localidades de São Paulo e do Paraná, usando os modelos DSSAT/OILCROP-SUN e FAO. Revista Brasileira de Agrometeorologia, v. 9, n. 1, p. 91-102, 2001.
SANTOS, R.D.; PEREIRA, L.G.R.; NEVES, A.L.A.; RODRIGUES, J.A.S.; COSTA, C.T.F.; OLIVEIRA, G.F. Agronomic characteristics of forage sorghum cultivars for silage production in the lower middle San Francisco Valley. Acta Scientiarum. Animal Sciences Journal. v. 35, n. 1, p. 13-19, 2013.

SOUZA, R. F ; BARROS, A. C.; BARROS, A. H. C.; TABOSA, J. N. Estimates for maize yield (Zea mays L.) in rainfed and irrigated crops determined by the method of agroecological ZONE/FAO (ZAE/FAO), state of Alagoas, Brazil. Revista Brasileira de Agricultura Irrigada, v. 8, n.2, p. 127-138, 2014.

SHRESTHA, N.; GEERTS, S.; RAES, D.; HOREMANS, S.; SOENTJENS, S.; MAUPAS, F.; CLOUET, P. Yield response of sugar beets to water stress under western European conditions. Agricultural Water Management, Amsterdam, v. 97, n.2, p. 346350, 2010.

TABOSA, J. N. Cultivo do Sorgo Granífero no Nordeste. In: Maria Elisa Ayres Guidetti Zagatto Paterniani; Aildson Pereira Duarte; Alfredo Tsunechiro. (Org.). Diversidade e Inovações na Cadeia Produtiva de Milho e Sorgo na era dos Transgênicos. 1ed. Campinas - SP: Instituto Agronômico de Campinas / Associação Brasileira de Milho e Sorgo, 2012, v. 1, P. 581-604.

TABOSA, J. N.; SIMPLÍCIO, J.B.; NASCIMENTO, M. M. A. do; REIS, O. V. dos; SILVA, F. G.da; LIMA, J. M. P. Comportamento de Cultivares de Sorgo Forrageiro em Diferentes Ambientes do SemiÁrido Nordestino. In: XXVII CONGRESSO NACIONAL DE MILHO E SORGO, 2008, LONDRINA. ANAIS CD- ROM DO XXVII CONGRESSO NACIONAL DE MUILHO E SORGO. LONDRINA: IAPAR / EMBRAPA, 2008. 